\title{
FASTER RENDERING OF LARGE PSEUDO-DYNAMIC SCENES USING HIERARCHICAL DYNAMIC LODS
}

\author{
Vitaly Semenov $^{1,2,3}$, Vasily Shutkin ${ }^{1}$ and Vladislav Zolotov ${ }^{1}$ \\ ${ }^{I}$ Ivannikov Institute for System Programming of the Russian Academy of Sciences \\ 25, Alexander Solzhenitsyn st., Moscow, 109004, Russia \\ ${ }^{2}$ Moscow Institute of Physics and Technology (National Research University) \\ 9 Institutskiy per., Dolgoprudny, Moscow Region, 141701, Russia \\ ${ }^{3}$ National Research University Higher School of Economics \\ 20 Myasnitskaya Ulitsa, Moscow, 101000, Russia
}

\begin{abstract}
Rendering of large dynamic scenes with a convincing level of realism is a challenging problem of computer graphics. One of the promising approaches to rendering such scenes is a simplification of models and the use of multiresolution series of models with levels of detail (LOD) appropriate for the current view-dependent context. Nowadays, different simplification techniques, including hierarchical level of details (HLOD), have been proposed and widely applied. However, these techniques face dramatic difficulties when scenes undergo modifications. Known attempts of their adaptation for dynamic scenes have not resulted in significant progress. In the paper an alternative approach to rendering pseudo-dynamic scenes is presented. Such scenes have numerous industrial applications, in particular, visual spatial-temporal modeling of complex construction projects, city infrastructure programs, and machinery assembly production. A concept of hierarchical dynamic levels of details (HDLOD) is introduced and complementary methods for generation of HDLODs and their visualization are proposed. The results of computational experiments are also presented.
\end{abstract}

\section{KEYWORDS}

Rendering, Dynamic Scenes, Polygonal Models, Level of Details

\section{INTRODUCTION}

In recent years, the problem of rendering of large dynamic scenes with a convincing level of realism has received increasing attention. Often such scenes are composed of thousands and millions of polygonal models created or acquired at a very high resolution level. Being scene objects, the models can be supplemented by dynamic behaviors assuming their appearance, movement or disappearance in accordance with a deterministic schedule or randomly occurring events.

Highly detailed models with accurately specified schedules are not always required, but prevent effective rendering of scenes by current graphics systems at reasonable interactive frame rates. This observation is critically important for many CAD/CAM/CAE applications whose functions involve both display of complex scenes and manipulation of individual objects in space and time dimensions.

One of the promising approaches to rendering complex scenes is a simplification of models and the use of multiresolution series of models with appropriate levels of detail (LOD) for the current view-dependent context. This research topic has a long history in computer graphics which began with the introduction of the LOD concept by James Clark in 1976. The concept realization assumes the appropriate LODs should be selected and rendered so that finer representations are used for the nearest objects and coarser approximations are applied to the farther objects. Although this concept allows broader interpretations, including simplifications of spatial-temporal models, the main efforts of researchers focused on polygonal meshes and sometimes on meshes with colors and textures.

Numerous simplification algorithms have been proposed following the same goal: to reduce the number of polygons of the mesh while preserving details and characteristics of the original as much as possible. Generally, the algorithms differ in the underlying decimation operation used to transform the mesh and the error metric used to measure the introduced geometric error. By decimation operations the algorithms can be 
categorized into the following classes: vertex removal, edge collapse, face collapse, vertex clustering and vertex merging. Vertex removal algorithms commonly use an iterative prioritization and retriangulation scheme within which the vertices are sequentially selected, their adjacent faces are removed and the resulting holes are retriangulated (Schroeder 1992; Soucy and Laurendeau 1996). Vertex clustering algorithms employ an auxiliary grid surrounding the simplified mesh. Within each cell, the cell's vertices are clustered together into a single vertex, and the incident faces are updated accordingly (Rossignac and Borrel 1993). Edge removal algorithms iteratively choose an edge to be contracted into a single point and degenerate the adjacent faces (Hoppe 1996; Ronfard and Rossignac 1996; Guéziec 1995). None of these algorithms provides the combination of efficiency, quality, and generality which would be desired for complex objects and scenes. We highlight the algorithm most relevant to our goals (Garland 1997). By leveraging both edge contraction, vertex aggregation and quadric error metrics, the algorithm exhibits good performance and quality for non-manifold and unconnected meshes.

The aforementioned algorithms help in the generation of LODs for single polygonal objects, but are quite limited to manage complex scenes. Nowadays, hierarchical level of details (HLOD) techniques are widely employed for such purposes (Erikson 2001). Unlike traditional ones, HLODs provide simplified representations not of the individual objects, but of the entire groups of objects organized into multilevel hierarchies. Instead of analyzing individual objects and displaying appropriate LODs, it becomes possible to render the entire groups of objects at once when traversing HLOD hierarchies and analyzing descending nodes. Processing of all individual objects is generally unnecessary.

However, HLODs face dramatic difficulties when scenes undergo modifications. Their representations should be recomputed whenever the objects are added, deleted or moved in the scene. The CPU time required for recomputations is typically greater than that for rendering the scene on GPU, which makes HLODs useless for dynamic scenes. Known attempts to optimize HLODs by using incremental updates and their asynchronous parallel execution on shared memory multiprocessor systems, haven't resulted in significant success (Erikson 2001).

In the paper we present an alternative approach to rendering large dynamic scenes for which the following two assumptions are made. The first assumption concerns the deterministic nature of the events in the scene, which allows you to analyze them before modeling the scene. The second assumption concerns the dominant number of events associated with the appearance and removal of objects relative to the number of the object movements. We call such scenes pseudo-dynamic ones and claim a large number of industrial applications that operate with just such scenes. In particular, these are visual planning and modeling of complex construction projects, city infrastructure programs, and machinery assembly production.

We also propose hierarchical dynamic levels of details (HDLOD) for rendering such scenes and specify an algorithm for generation of HDLODs. The results of preliminary computational experiments are also presented in the paper.

\section{HIERARCHICAL DYNAMIC LODS}

Let the scene $S(t)$ be defined in three-dimensional Euclidian space $E^{3}$ on the model time period $t \in[0, T]$ and be represented as a flat composition of objects $s\left(g_{s}, f_{s}\right) \in S$ having fixed spatial positions and unchangeable geometry representations $g_{s} \subseteq E^{3}$. The presence status of objects and their groups in the scene is determined by the behavior functions $f_{s}(t):[0, T] \rightarrow\{0,1\}$ so that the function $f_{s}(t)$ takes the unit value if the object $s$ is present in the scene at the time $t$ and the zero value if the object is absent. Throughout the work we call such scenes deterministic pseudo-dynamic. It is worth mentioning that continuously moving objects can be modelled through the discretization of a continuous path and simulation of successive events of the appearance and removal of object instances along this path. We suggest that relatively few objects are continuously moved or even that such movements are completely absent in the scene.

Contrary to this limitation, the considered class of scenes has numerous industrial applications. Figure 1 shows an example of a scene simulating the construction of a stadium in accordance with a preliminary prepared project schedule. As modeling time changes, building elements and pieces of equipment are mounted, installed on a construction site or removed. The landscape elements remain unchanged throughout the entire modeling period. Such behaviors are reproduced by standard functions presented in Figure 2. 

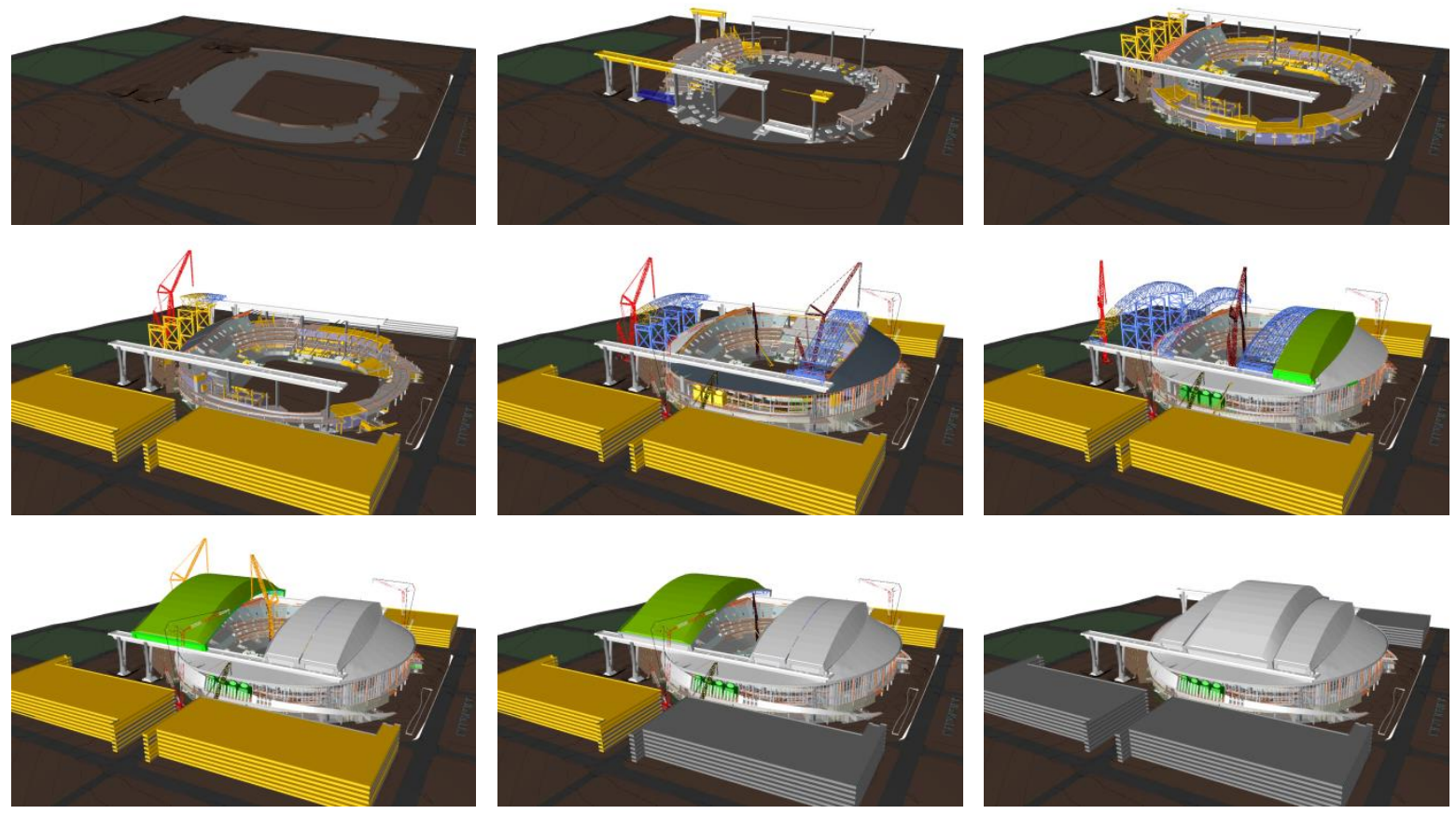

Figure 1. Example of a pseudo-dynamic scene simulating the construction of a stadium

In the following discussion we assume that the scene objects are geometrically represented as triangle sets with vertices referring to common set of points. No assumptions about solidness of the objects and manifoldness of their boundary representations are made because often only "polygon soups" are provided for rendering purposes without any guarantees of regular topological properties.

We also do not impose any restrictions on the coherence of the scenes, although we expect that in the industrial project scenes with proper organization of workflows this factor can be relatively high. By spatialtemporal coherence $\sigma$ we mean the difference $1-\rho$ where $\rho$ is the average distance between objects participating in subsequent events related to the size of the entire scene. Being defined in this way, the introduced coherence factor lies in the following range: $0 \leq \sigma \leq 1$.

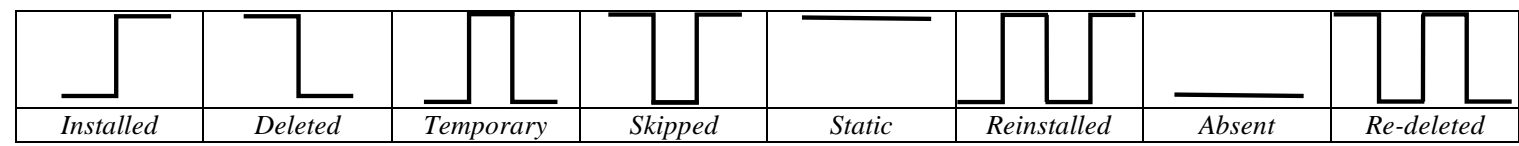

Figure 2. Some typical behavior functions

We will call the HDLOD a cluster tree $C(G, F)=\left\{c\left(g_{c}, f_{c}\right), \prec\right\}$ represented by a set of clusters $c\left(g_{c}, f_{c}\right)$ with prescribed geometry representations $g_{c}$ and behavior functions $f_{c}(t):[0, T] \rightarrow[0,1]$. Unlike the object functions $f_{s}(t)$, which take values 0 or 1 , the cluster functions $f_{c}(t)$ take values in the range between 0 and 1 thereby employing the concept of partial truth. Indeed, since some of the cluster objects may be present in the scene at some time point, while others may be absent, an unambiguous verdict on the presence status of the entire cluster cannot be made. For the clusters an agglomerative relation $\prec$ is also defined so that $c^{\prime} \prec c$ only if the cluster $c^{\prime} \in C$ is a direct child of the parent cluster $c \in C$. The leaves of the tree are the precisely defined individual objects. Interior nodes represent clusters aggregating the geometries and behaviors of the objects in corresponding subtrees. The root is the cluster containing the least accurate and most simplified representation of the entire scene. No assumptions are made about the degree of nodes and the balance of the tree. Figure 3 provides an example of unbalanced HDLOD tree with the nodes of varied degree. 
Each cluster $c \in C$ stores not only the geometry and behavior representations but also such derived attributes as axis-aligned or oriented bounding box $b_{c}$, size or strength $w_{c}$, spatial tolerance $\varepsilon_{c}$, temporal tolerance $\gamma_{c}$ as defined below. Basically, these attributes are computed when generating HDLODs and are applied when displaying HDLODs. Thus, each HDLOD cluster is represented as a tuple $c\left(g_{c}, f_{c}, b_{c}, w_{c}, \varepsilon_{c}, \gamma_{c}\right)$.

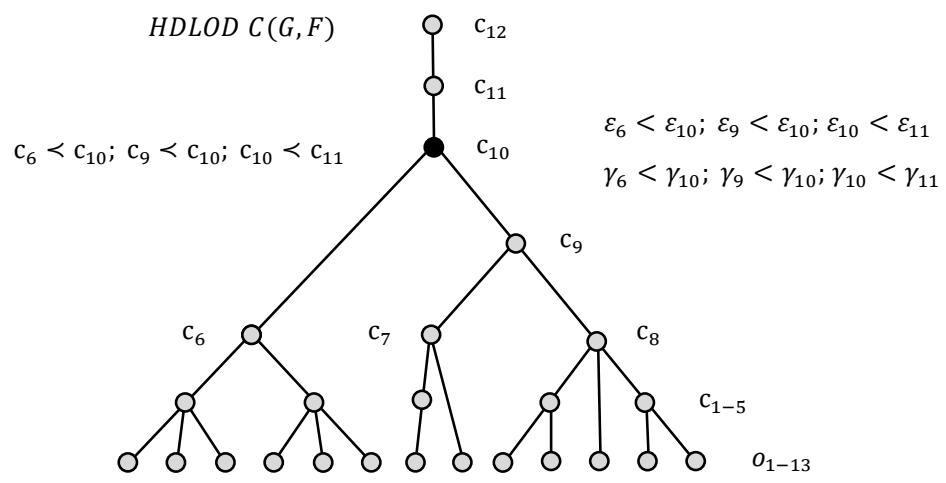

Figure 3. HDLOD tree and some agglomerative and algebraic relations

Let us dwell on the aforementioned parameters in more detail since they are important for displaying scenes with a given accuracy. Although these parameters allow different interpretations, we define $\varepsilon_{c}$ as an absolute tolerance which establishes the possible maximum local deviation of the geometric representation of the cluster $c$ from an aggregated representation of the originating objects $o \prec . .<c^{\prime} \prec c$ :

$$
\varepsilon_{c}=\max _{c^{\prime}<c}\left(\varepsilon_{c^{\prime}}\right)+D_{H}\left(g_{c}, \bigcup_{c^{\prime}<c} g_{c^{\prime}}\right)
$$

Here, the tolerance $\varepsilon_{c}$ is recurrently defined using the Hausdorff distance $D_{H}(A, B)$ that is the greatest of all the distances from a point in one set to the closest point in the other set:

$$
\left.D_{H}(A, B)=\max _{2} \max _{x \in A} \min _{y \in B} D(x, y), \max _{y \in B} \min _{x \in A} D(x, y)\right\},
$$

where $A, B$ are closed sets of points and $D(x, y)$ is a metric function in Euclidian space.

The temporal tolerance $\gamma_{c}$ is defined as a maximum deviation of the cluster behavior from individual behaviors of the originating objects:

$$
\gamma_{c}=\max _{c^{\prime}<c}\left(\gamma_{c^{\prime}}+D_{F}\left(f_{c}, f_{c^{\prime}}\right)\right) \text {, }
$$

where the distance $D_{F}\left(f_{A}, f_{B}\right)$ is determined using the functional metric:

$$
D_{F}\left(f_{A}(t), f_{B}(t)\right)=\frac{1}{T} \int_{0}^{T}\left|f_{A}(t)-f_{B}(t)\right| d t
$$

Thus, the introduced parameters are used to estimate the spatial proximity of objects, the geometry deviations and the proximity of temporal behaviors. The spatial tolerances are evaluated during the geometry simplification using one of the methods mentioned in the introduction. The temporal tolerances should be evaluated simultaneously with the simplification of behavioral functions. We propose the following computation method:

$$
f_{c}(t)=\frac{\sum_{c^{\prime<c}} w_{c^{\prime}} f_{c^{\prime}}(t)}{\sum_{c^{\prime}<c} w_{c^{\prime}}}
$$

The use of the weighted sums enables us to take into account the behavior of significant objects to a greater degree and to reproduce the cluster behavior in the most relevant way. If the function $f_{c}(t)$ takes the unit value then all the cluster objects are present in the scene at the time $t$ and the cluster should be displayed as a simple object. If the function takes the zero value then all the objects are absent at the time $t$ and should be ignored completely. Otherwise, the spatial time-dependent tolerance (spatial error induced by both geometrical and temporal simplifications) should be estimated to decide whether to display the cluster or to ignore the cluster or to use more accurate descending representations. The resulting time-dependent spatial tolerance $\delta_{c}(t)$ can be directly expressed through the cluster behavior function as follows: 


$$
\delta_{c}(t)=\left\{\begin{array}{c}
0, \quad \text { if } f_{c}(t)=0 \\
\varepsilon_{c}, \quad \text { if } f_{c}(t)=1 \\
\varepsilon_{c}+\left(1-f_{c}(t)\right) \sum_{c^{\prime}<c} w_{c^{\prime}}, \quad \text { if } \frac{1}{2} \leq f_{c}(t)<1 \\
\varepsilon_{c}+f_{c}(t) \sum_{c^{\prime}<c} w_{c^{\prime}}, \quad \text { if } 0<f_{c}(t)<\frac{1}{2}
\end{array}\right.
$$

When visualizing a scene, the cluster tree is traversed starting from the root and descending to the leaves. At each node the cluster attributes are analyzed to determine the necessity of the further traversal of the subtree. It is checked whether the cluster is present in the scene at a specified model time, whether the cluster bounding box falls into the view frustum, and whether the tolerances are small enough to obtain the required resolution. If all the conditions are satisfied, the cluster representation is applied for rendering immediately. In such case, a whole subtree can be pruned from the traversal, which decreases the number of visualized objects and accelerates the rendering process. If the third condition is not met, the traversal of the cluster subtree continues and descending nodes are underwent to similar checks until the leaves with precisely given scene objects are reached.

Pseudocode of the specified algorithm is presented in Figure 4. The main procedure DISPLAY invokes DISPLAY CLUSTER for the root cluster with the given parameters of camera view, current modeling time and graphics system resolution. This procedure in turns traverses descending nodes by calling itself recursively. The visited clusters are rendered with the required accuracy when it becomes possible and reasonable. The purpose and use of auxiliary functions are quite transparent.

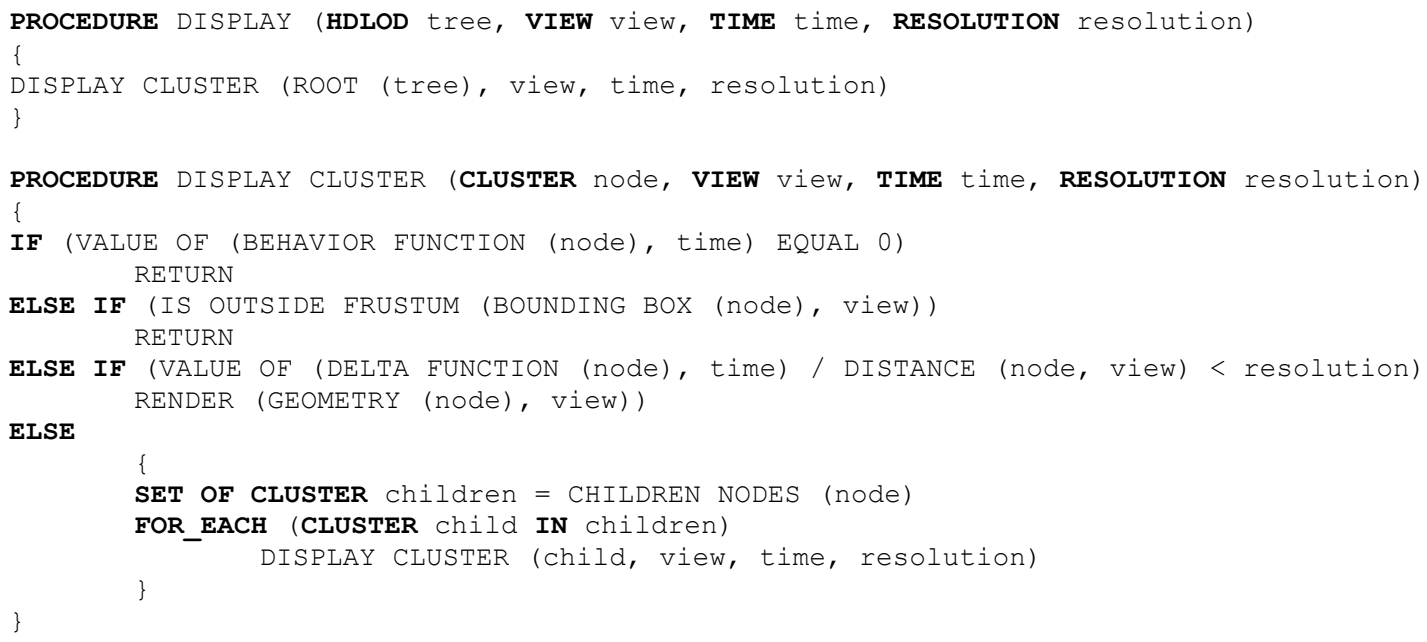

Figure 4. Pseudocode for displaying HDLODs

\section{HDLODS GENERATION}

The HDLODs can be generated automatically using the proposed method that leverages both hierarchical (bottom-up) clustering and multi-level accuracy control. The method is effective for both view-dependent rendering and view-frustum culling purposes. The method starts with the individual objects and progressively groups these together into larger and larger clusters until the desired number of levels is achieved. The root clusters can be further simplified as a series of LODs if it is planned to render the scene as part of a more complex composition. Clustering should be carried out under strict control of accuracy, and the resulting HDLOD tree should meet numerous requirements and conditions regarding the expected number of levels of details, the degree of nodes, the spatial density and overlap of sibling clusters, the temporal proximity of sibling clusters, and the descent of the cluster complexity with increasing levels. 
Unfortunately, classical clustering methods cannot be directly applied to the HDLOD generation problems. The imposed requirements are quite complex and can contradict to each other. That hinders the mathematical formalization of metric functions and linkage criteria necessary for the clustering methods (Xu 2015). Unacceptably high computational complexity of the methods is another reason preventing the adaption of classical results.

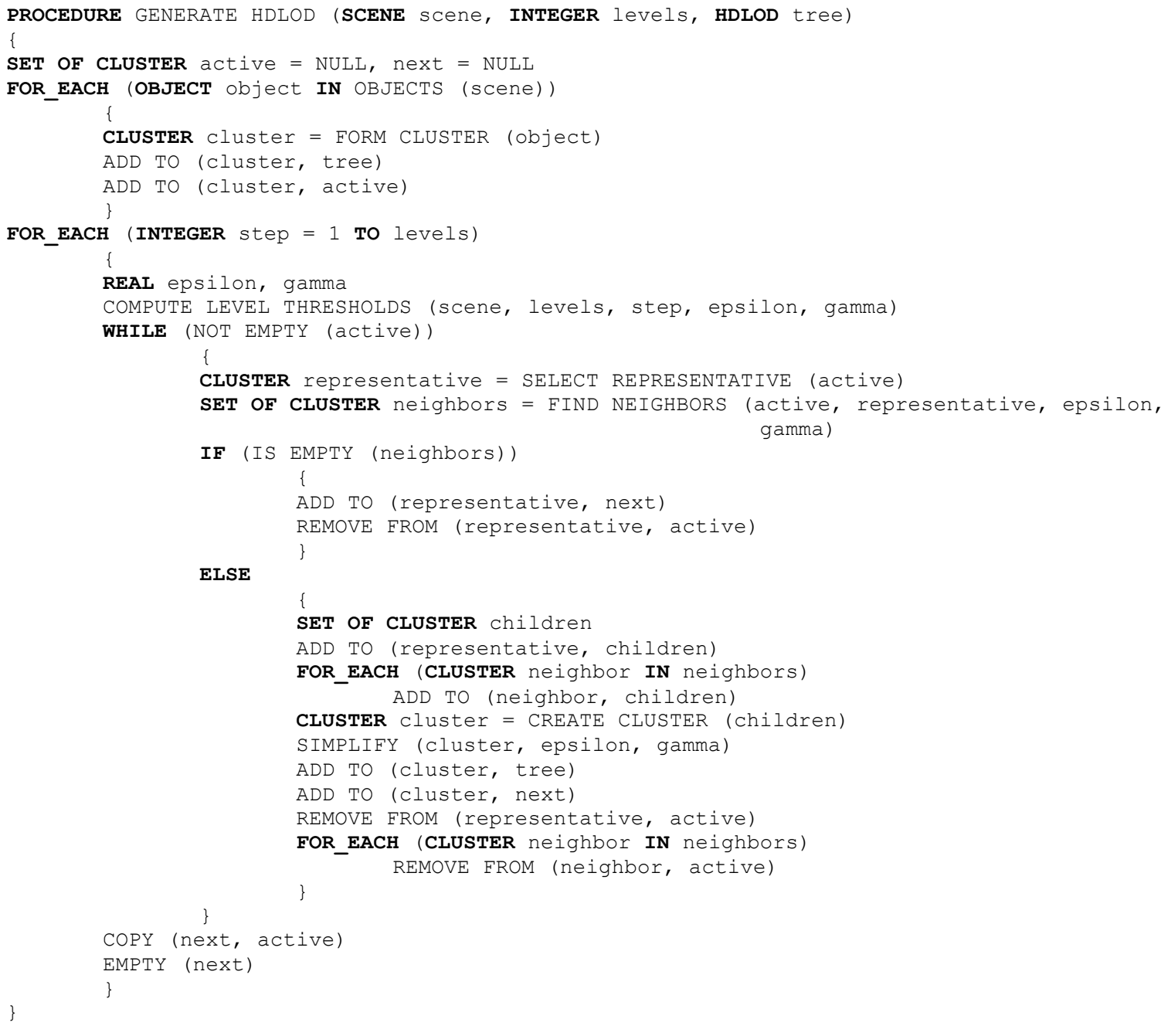

Figure 5. Pseudocode for generating HDLODs

For example, a naïve implementation of agglomerative clustering has a time complexity of $O\left(n^{3}\right)$ and requires $O\left(n^{3}\right)$ memory, which makes it useless for even simple scenes. Faster hierarchical clustering with a time complexity of $O\left(n^{2}\right)$ and memory consumption $O\left(n^{2}\right)$ is also not feasible for the problems discussed (Xu 2015).

Therefore, we prefer to form HDLOD trees by employing other principles. The clustering process is divided into steps, at each of which the formed clusters satisfy certain requirements for accuracy. As new steps are taken, the requirements are weakened in such a way that the process is guaranteed to terminate after a specified number of steps equal to the number of levels of details $L$. Only active clusters are involved in the clustering process as opposed to passive clusters that have already been analyzed and grouped.

At each planned step $l$ of the method $(1 \leq l \leq L)$, an attempt to form new clusters with the tolerances $\varepsilon_{c} \leq \varepsilon(l), \gamma_{c} \leq \gamma(l)$ is made. For this purpose threshold values $\varepsilon(l), \gamma(l)$ are preliminary computed in such a way that the thresholds increase monotonously with increasing levels $\varepsilon(l-1)<\varepsilon(l), \gamma(l-1)<\gamma(l)$. For example, the tolerance thresholds can be chosen as follows:

$$
\varepsilon(l)=2 w l / R, \quad \gamma(l)=2 \tau l,
$$


where $w$ is the size of the smallest scene object related to the size of the entire scene (e.g. diagonal of the box bounding all the scene objects over the modeling period, including temporally installed and removed ones), $R$ stands for the effective display resolution (the typical number of pixels along one of the axes divided by the camera field of view) and $\tau$ is the effective temporal discretization used for the animation purposes (the typical time step between successive events related to the entire modeling period).

Once the tolerance thresholds have been determined, clusters can be formed step by step. At each planned step, representatives among active clusters are selected using space-filling curves and corresponding orderings by Hilbert (Samet 2006). On the one hand, it allows representatives to be chosen throughout the entire volume of the scene, and on the other hand, to localize them in densely filled regions. Then, for each representative the neighbors are sought in both spatial and temporal dimensions. The neighbors should satisfy the conditions of spatial and temporal proximity as well as ensure the formation of parent clusters with the planned accuracy. If no proper neighbors are found for the selected representative, it is excluded from the analysis at the current step, but participates in the succeeding cluster formation. The parent cluster behavior is determined using computations of the weighted behavior function as specified above. The resulting geometry representation of the parent cluster is merged from polygonal models of the originating clusters and then is simplified using the highlighted algorithm (Garland 1997). Pseudocode of the specified algorithm is presented in Figure 5.

Using indices preliminary computed and deployed, clustering can be done in $O(n \log n)$, where $n$ is the number of the scene objects. This is a promising result compared to the classical clustering methods mentioned above. Although many different techniques can be used for spatial-temporal indexing (Samet 2006), in particular, regular dynamic octrees exhibiting good performance for pseudo-dynamic scenes (Morozov 2018), we utilized simple indexing structures to search for objects (more exactly, centers of their bounding boxes) in each dimension separately.

\section{COMPUTATIONAL EXPERIMENTS}

To validate the introduced HDLODs concept and the proposed method for their automatic generation and visualization, a series of computational experiments has been carried out. Display frame times were measured when navigating across a given scene at fixed modeling times and when animating and viewing the scene from fixed camera positions throughout the modeling period. As a benchmark, the presented in Figure 1 dynamic scene of the stadium construction was explored. The stadium model consisted of 52630 building elements represented by polygonal meshes totally containing 9799257 triangles. The construction schedule consisted of 48902 activities each of which was responsible for certain works on the construction site and induced related events in the dynamic scene. The spatial-temporal coherence factor $\sigma$ for the scene was evaluated and amounted to 0.78 . This factor is usually quite high for typical industrial scenes and leads to higher performance of visualization facilities.

To evaluate the effectiveness of the HDLODs with eliminated frustum culling factor, the experiments were repeated for a camera located at different distances from the scene. In the first position, the camera was placed at the closest distance, at which the entire scene was visible. In other positions, the camera was placed at the distances, at which the scene was occupying one-fourth and one-sixteenth of the screen area correspondingly. The times were chosen at the start and end of the modeling period as well as on one third and two thirds of the modeling period. The computational experiments have been carried out on a typical hardware configuration: Intel Core i7-4790 CPU (3.6 GHz), 16 GB of RAM, GeForce GTX $750 \mathrm{Ti}$ (2 GB).

Table 1 showcases the rendering performance results obtained during the specified experiments. The last column provides the results obtained using direct rendering methods without HDLODs. As seen, the use of HDLODs significantly increases the rendering performance compared to traditional techniques. The farther from the scene the camera is placed, the greater is the achieved effect. It is exhibited both when navigating the static scene fixed at the chosen times, and when animating the scene. It is worth mentioning that the dependence is super-linear and this gives reason to make conclusions about the scalability of the method with respect to the complexity of both static and dynamic scenes. Compared to traditional HLODs which would lead to excessive computing costs for updating, even with minor local changes in the scene, HDLODs are prepared beforehand and do not require any updates when animating the scene throughout the entire modeling period. 
Table 1. Frame times (in milliseconds) during the visualization of the stadium construction scene

\begin{tabular}{lllll}
\hline & $\mathbf{1 / 1}$ screen view & $\mathbf{1 / 4}$ screen view & $\mathbf{1 / 1 6}$ screen view & No HDLODs \\
\hline Start time & 0.37 & 0.32 & 0.31 & 26.81 \\
$1 / 3$ of the period & 7.17 & 3.31 & 1.38 & 31.36 \\
$2 / 3$ of the period & 21.9 & 7.35 & 4.4 & 124.8 \\
End time & 1.73 & 0.79 & 0.6 & 142.4 \\
Animation & 20.01 & 12.91 & 6.63 & 86.35 \\
\hline
\end{tabular}

\section{CONCLUSION}

Thus, the concept of hierarchical dynamic levels of details has been introduced and the complementary methods for their automatic generation and visualization have been proposed in the paper. As opposed to traditional LODs techniques suitable only for static scenes, the developed methods are applicable to a wide class of deterministic pseudo-dynamic scenes arising in numerous industrial applications, including visual modeling of complex construction projects, city infrastructure programs, and machinery assembly production. The results of conducted computational experiments have confirmed high efficiency of HDLODs. Future research will focus on different algorithmic options for the developed methods, as well as on generalizations necessary for applying to scenes with continuously moving objects and objects with complex materials.

\section{REFERENCES}

Erikson, C. et al., 2001. HLODs for Faster Display of Large Static and Dynamic Environments. I3D '01 Proceedings of the 2001 symposium on Interactive 3D graphics. New York, USA, pp. 111-120.

Garland, M. and Heckbert, P.S., 1997. Surface simplification using quadric error metrics. SIGGRAPH '97 Proceedings of the 24th annual conference on Computer graphics and interactive techniques. New York, USA, pp. 209-216.

Guéziec, A., 1995. Surface Simplification With Variable Tolerance. 2nd Annual International Symposium on Medical Robotics and Computer Assisted Surgery, Baltimore, USA, pp. 132-139.

Hoppe, H., 1996. Progressive Meshes. SIGGRAPH '96 Proceedings of the 23rd annual conference on Computer graphics and interactive techniques. New York, USA, pp. 99-108.

Morozov, S. et al, 2018. Indexing of Hierarchically Organized Spatial-Temporal Data Using Dynamic Regular Octrees. In: Petrenko A., Voronkov A. (eds) Perspectives of System Informatics. PSI 2017. Lecture Notes in Computer Science, vol 10742, pp. 276-290.

Ronfard, R. and Rossignac, J., 1996. Full-range approximation of triangulated polyhedra. In Computer Graphics Forum, Vol. 15, No. 3, pp. 67-76.

Rossignac J. and Borrel P., 1993. Multi-resolution 3D approximations for rendering complex scenes. In: Falcidieno B., Kunii T.L. (eds) Modeling in Computer Graphics. IFIP Series on Computer Graphics. Springer, Berlin, Heidelberg, Germany.

Samet, H., 2006. Foundations of Multidimensional and Metric Data Structures. Morgan Kaufmann, Burlington, USA.

Schroeder, W.J. et al., 1992. Decimation of Triangle Meshes. SIGGRAPH '92 Proceedings of the 19th annual conference on Computer graphics and interactive techniques. New York, USA, pp. 65-70.

Soucy, M. and Laurendeau, D., 1996. Multiresolution Surface Modeling Based on Hierarchical Triangulation. In Computer Vision and Image Understanding, Vol. 63, No. 1, pp. 1-14.

Xu, D. and Tian, Y., 2015. A Comprehensive Survey of Clustering Algorithms. In Annals of Data Science, Vol. 2, pp. 165-193. 